\title{
(NON)REGULARITY OF PROJECTIONS OF MEASURES INVARIANT UNDER GEODESIC FLOW
}

\author{
ESA JÄRVENPÄÄ ${ }^{1}$, MAARIT JÄRVENPÄ $\ddot{A}^{2}$, AND MIKA LEIKAS ${ }^{3}$
}

\begin{abstract}
We show that, unlike in the 2-dimensional case [LL], the Hausdorff dimension of a measure invariant under the geodesic flow is not necessarily preserved under the projection from the unit tangent bundle onto the base manifold if the base manifold is at least 3-dimensional. In the 2-dimensional case we reprove the preservation theorem due to Ledrappier and Lindenstrauss [LL] using the general projection formalism of Peres and Schlag [PS]. The novelty of our proof is that it illustrates the reason behind the failure of the preservation in higher dimensional case. Finally, we show that the projected measure has fractional derivatives of order $\gamma$ for all $\gamma<(\alpha-2) / 2$ provided that the invariant measure has finite $\alpha$-energy for some $\alpha>2$ and the base manifold has dimension 2 .
\end{abstract}

\section{INTRODUCTION}

The study of the behaviour of Hausdorff dimension under projectiontype mappings dates back to the 1950's when Marstrand [Mar] proved a well-known theorem according to which the Hausdorff dimension of a planar set is preserved under typical orthogonal projections. In $[\mathrm{K}]$ Kaufman verified the same result using potential theoretical methods, and in [Mat1] Mattila generalized it to higher dimensions. For measures the analogous principle, discovered by Kaufman [K], Mattila [Mat2], $\mathrm{Hu}$ and Taylor [HT], and Falconer and Mattila [FM], can be stated in the following form: Let $m$ and $n$ be integers such that $0<m<n$ and let $\mu_{V}$ be the image of a compactly supported Radon measure $\mu$ on $\mathbb{R}^{n}$ under the orthogonal projection onto an $m$-plane $V$. Then for almost all $m$-planes $V$ we have

$$
\operatorname{dim}_{\mathrm{H}} \mu_{V}=\operatorname{dim}_{\mathrm{H}} \mu \text { provided that } \operatorname{dim}_{\mathrm{H}} \mu \leq m .
$$

On the other hand, for almost all $m$-planes $V$

$$
\mu_{V} \ll \mathcal{L}^{m} \text { provided that } \operatorname{dim}_{\mathrm{H}} \mu>m \text {. }
$$

2000 Mathematics Subject Classification. 58C35, 53D25, 37D40, 37A05.

Key words and phrases. Invariant measure, geodesic flow, projection, preservation of Hausdorff dimension.

MJ and ML acknowledge the support of the Academy of Finland, project \#48557. 
(Above $\operatorname{dim}_{\mathrm{H}}$ is Hausdorff dimension, $\mathcal{L}^{m}$ is Lebesgue measure, and the symbol « denotes the absolute continuity.) In the case that $\mu$ has finite $m$-energy a substantially stronger form of (1.2) holds: we have for all typical $m$-planes that

$$
\mu_{V} \ll \mathcal{L}^{m} \text { with Radon-Nikodym derivative in } L^{2} \text {. }
$$

Analogies of these results have been investigated for typical smooth mappings in the sense of prevalence and for infinite dimensional spaces in [SY], [HK1], and [HK2]. In [PS] Peres and Schlag extended (1.1), (1.2), and (1.3) to Sobolev dimensions of measures on compact metric spaces and parametrized families of transversal mappings in an elegant way. For the purposes of the present paper, a significant difference between the earlier results and those of [PS] is that Peres and Schlag generalized (1.3) in terms of fractional derivatives by showing that if the original measure has finite $(m+\varepsilon)$-energy, then densities of typical projections onto $m$-dimensional spaces have fractional derivatives of order $\varepsilon / 2$ in $L^{2}$. For more detailed information about a variety of related contributions, see [Mat4] and [PS].

In this paper we address the question of studying measures on Riemannian manifolds which are invariant under the geodesic flow. Although they are measures on the unit tangent bundle of the manifold, it is natural to try to describe their dimensional properties on the base manifold. This can be done using the natural projection from the unit tangent bundle onto the manifold. (For a discussion of connections to the Besicovitch-Kakeya problem, see [LL].) Even though the above mentioned results (1.1), (1.2), and (1.3) are genuinely "almost all"results, meaning that they do not provide information about any specified projection, similar methods work for the natural projection from the unit tangent bundle onto the Riemannian surface. This interesting feature was discovered quite recently by Ledrappier and Lindenstrauss in [LL].

Theorem 1.1 (Ledrappier, Lindenstrauss). Let $M$ be a compact Riemannian surface, let $\mu$ be a Radon probability measure on the unit tangent bundle $S M$, and let $\Pi: S M \rightarrow M$ be the natural projection. Assuming that $\mu$ is invariant under the geodesic flow, the following properties hold for the image $\Pi_{*} \mu$ of $\mu$ under $\Pi$ :

(1) If $\operatorname{dim}_{\mathrm{H}} \mu \leq 2$, then $\operatorname{dim}_{\mathrm{H}} \Pi_{*} \mu=\operatorname{dim}_{\mathrm{H}} \mu$.

(2) If $\operatorname{dim}_{\mathrm{H}} \mu>2$, then $\Pi_{*} \mu \ll \mathcal{L}^{2}$.

Analogously to (1.3), Ledrappier and Lindenstrauss proved that if $\mu$ has finite $\alpha$-energy for $\alpha>2$, then the Radon-Nikodym derivative is a $L^{2}$-function. They also addressed the question of whether this could be further generalized in terms of fractional derivatives. In addition to giving a positive answer to this question by employing the techniques from [PS], we consider another issue brought up in [LL] which is the 
validity of Theorem 1.1 for higher dimensional base manifolds. Quite surprisingly, it appears that the Hausdorff dimension is not necessarily preserved. Recalling the case of (1.1), (1.2), and (1.3), one might first think that the generalization from dimension 2 to higher dimensions is a question of finding correct methods. However, in section 4 we give a new proof for Theorem 1.1 which explains why the preservation fails in higher dimensions.

This paper is organized as follows: In section 2 we discuss the general projection formalism of Peres and Schlag [PS] which plays an important rôle in this work, whereas in section 3 we recall the basic assumptions from [LL] and introduce our setting. The main part of section 4 is devoted to proving that the parametrized family of mappings we are working with is transversal (Proposition 4.1). Then we apply the machinery of $[\mathrm{PS}]$ and a result from [JJL] to reprove Theorem 1.1, and explain why this does not work for higher dimensional base manifolds (Remark 4.5). The question concerning the fractional derivatives of the density of the projected measure will be dealt with in section 5 . We prove that if the $\alpha$-energy of $\mu$ is finite for some $\alpha>2$, then $\Pi_{*} \mu$ has fractional derivatives of order $\gamma$ in $L^{2}$ for all $\gamma<(\alpha-2) / 2$ (Theorem 5.1). Finally, in the last section we give examples of higher dimensional manifolds and invariant measures on the unit tangent bundles whose Hausdorff dimensions decrease when projecting onto the base manifolds. Remark 4.5 gives a base for constructing such examples.

\section{General projection formalism of Peres and Schlag}

In this section we recall the notation and results we need from [PS]. Given $\gamma \geq 0$, let $\|\nu\|_{2, \gamma}$ be the Sobolev norm of a finite Borel measure $\nu$ on $\mathbb{R}^{n}$, that is,

$$
\|\nu\|_{2, \gamma}=\left(\int|\hat{\nu}(\xi)|^{2}|\xi|^{2 \gamma} d \mathcal{L}^{n}(\xi)\right)^{1 / 2}
$$

where

$$
\hat{\nu}(\xi)=\int e^{-i \xi \cdot x} d \nu(x)
$$

is the Fourier transform of $\nu$. The Sobolev dimension of $\nu$ is

$$
\operatorname{dim}_{\mathrm{S}} \nu=\sup \left\{\left.\alpha \in \mathbb{R}\left|\int\right| \hat{\nu}(\xi)\right|^{2}(1+|\xi|)^{\alpha-1} d \mathcal{L}^{d}(\xi)<\infty\right\} .
$$

Given $\alpha \geq 0$, the $\alpha$-energy of a finite Borel measure $\nu$ on a compact metric space $(Y, d)$ is denoted by $I_{\alpha}(\nu)$, that is,

$$
I_{\alpha}(\nu)=\int_{Y} \int_{Y} d(x, y)^{-\alpha} d \nu(x) d \nu(y) .
$$

For the rest of this section, we restrict our consideration to the 1dimensional parameter space. 
Basic assumptions. Let $(Y, \mathrm{~d})$ be a compact metric space, let $J \subset \mathbb{R}$ be an open interval, and let $P: J \times Y \rightarrow \mathbb{R}$ be a continuous function. Assume that for any $l=0,1, \ldots$ there is a constant $\widetilde{C}_{l} \geq 1$ such that

$$
\left|\partial_{t}^{l} P(t, y)\right| \leq \widetilde{C}_{l}
$$

for all $t \in J$ and $y \in Y$.

For all $t \in J$ and $x, y \in Y$ with $x \neq y$, define

$$
T_{t}(x, y)=\frac{P(t, x)-P(t, y)}{\mathrm{d}(x, y)} .
$$

We assume that the following form of transversality holds: there is a constant $C_{T}$ such that for all $t \in J$ and for all $x, y \in Y$ with $x \neq y$ the condition $\left|T_{t}(x, y)\right| \leq C_{T}$ implies that

$$
\left|\partial_{t} T_{t}(x, y)\right| \geq C_{T}
$$

In addition, the function $T_{t}$ is assumed to be regular in the following sense: For all $l=0,1, \ldots$ there exists a constant $C_{l}$ such that

$$
\left|\partial_{t}^{l} T_{t}(x, y)\right| \leq C_{l}
$$

for all $t \in J$ and $x, y \in Y$ with $x \neq y$.

In the following theorem from [PS], which serves as a significant tool in Proposition 4.2, we use the notation $P_{t}(\cdot)=P(t, \cdot)$. Moreover, we denote by $f_{*} \mu$ the image of a measure on $\mu$ on $X$ under a mapping $f: X \rightarrow Z$ defined as $f_{*} \mu(A)=\mu\left(f^{-1}(A)\right)$ for all $A \subset Z$.

Theorem 2.1. Suppose that the assumptions (2.1), (2.3), and (2.4) are satisfied. Let $\alpha>0$ and let $\nu$ be a finite Borel measure on $Y$ such that $I_{\alpha}(\nu)<\infty$. Then there is a constant $C_{\gamma}$ such that

$$
\int_{J}\left\|\left(P_{t}\right)_{*} \nu\right\|_{2, \gamma}^{2} d t \leq C_{\gamma} I_{\alpha}(\nu)
$$

provided that $0<1+2 \gamma \leq \alpha$. Moreover, for any $\sigma \in(0, \min \{\alpha, 1\}]$ we have

$$
\operatorname{dim}_{\mathrm{H}}\left\{t \in J \mid \operatorname{dim}_{\mathrm{S}}\left(\left(P_{t}\right)_{*} \nu\right) \leq \sigma\right\} \leq 1+\sigma-\alpha .
$$

Proof. See [PS, Theorem 2.8].

We complete this section by stating a technical lemma which plays an important rôle in relating our setting to that of [PS].

Lemma 2.2. For all $t \in(0,1)$, let $\nu_{t}$ be a compactly supported Radon measure on $\mathbb{R}$. Suppose that $\mu$ is a Radon measure on $\mathbb{R} \times(0,1)$ such that for all Borel functions $g: \mathbb{R} \times(0,1) \rightarrow \mathbb{R}$

$$
\int g(x, t) d \mu(x, t)=\iint g(x, t) d \nu_{t}(x) d \mathcal{L}^{1}(t) .
$$

Assume that there is $\alpha>0$ such that $\operatorname{dim}_{\mathrm{H}} \nu_{t} \geq \alpha$ for $\mathcal{L}^{1}$-almost all $t \in(0,1)$. Then $\operatorname{dim}_{\mathrm{H}} \mu \geq 1+\alpha$. 
Proof. The proof of [JJL, Lemma 3.4] goes through in our setting. One simply needs to replace in the proof of [JJL, Lemma 3.4] the assumption according to which $I_{\alpha}\left(\nu_{t}\right)<\infty$ for all $t$ by the weaker one of Lemma 2.2 .

\section{Notation}

In this section, we define a transversal mapping appropriate to the setting of section 2. Our notation is similar to that in [LL]. Assume that $M$ is a smooth compact 2-dimensional Riemannian manifold. Denoting by $S M$ the unit tangent bundle, let $\mu$ be a Radon probability measure on $S M$ which is invariant under the geodesic flow, and let $\Pi: S M \rightarrow M$ be the natural projection.

Taking $p_{1}, p_{2} \in M$ sufficiently close to each other, we denote by $\gamma_{p_{1}, p_{2}}$ the unique shortest geodesic, parametrized by the Riemannian arc length, which connects $p_{1}$ and $p_{2}$, that is,

$$
\gamma_{p_{1}, p_{2}}(0)=p_{1} \text { and } \gamma_{p_{1}, p_{2}}\left(\mathrm{~d}_{M}\left(p_{1}, p_{2}\right)\right)=p_{2} \text {. }
$$

Here $\mathrm{d}_{M}$ is the distance induced by the Riemannian metric.

Basic assumptions. Let $I=[0,1]$. We choose an open set $U \subset M$ and a chart $\Phi: U \rightarrow \mathbb{R}^{2}$ with the following properties:

(1) $I^{2} \subset \Phi(U)$.

(2) Defining

$$
\mathcal{C}_{1}=\Phi^{-1}(I \times\{0\}) \text { and } \mathcal{C}_{2}:=\Phi^{-1}(I \times\{1\})
$$

and picking any $c_{1} \in \mathcal{C}_{1}$ and $c_{2} \in \mathcal{C}_{2}$, there exists a unique geodesic $\gamma_{c_{1}, c_{2}}$ connecting $c_{1}$ and $c_{2}$ such that its image $\Phi\left(\gamma_{c_{1}, c_{2}}(t)\right)=$ $\left(x_{1}(t), x_{2}(t)\right)$ satisfies

$$
\left|x_{1}^{\prime}(t)\right| \leq C\left|x_{2}^{\prime}(t)\right|
$$

for some $C>0$ for all $t \in\left[0, \mathrm{~d}_{M}\left(c_{1}, c_{2}\right)\right]$. Thus the tangents of the (images of) geodesics are uniformly bounded away from being horizontal. Further, $U$ is assumed to be so small that geodesics are close to straight lines. (We use scaled normal coordinates around a fixed point $m \in U$ with $\Phi(m)=(1 / 2,1 / 2)$.)

(3) Denoting by $\Gamma_{1}:\left[0, t_{1}\right] \rightarrow M$ and $\Gamma_{2}:\left[0, t_{2}\right] \rightarrow M$ the unique geodesics connecting the left-hand side end points of $\mathcal{C}_{1}$ and $\mathcal{C}_{2}$, and their right-hand side end points, respectively, we assume that $\Phi\left(\Gamma_{1}\right) \subset \Phi(U)$ and $\Phi\left(\Gamma_{1}\right) \subset \Phi(U)$.

As in [LL], we define a diffeomorphism $\Psi: I^{3} \rightarrow \Psi\left(I^{3}\right) \subset S M$ as follows:

$$
\Psi\left(y_{1}, y_{2}, t\right)=\left(\gamma_{p_{1}, p_{2}}\left(\mathrm{~d}_{M}\left(p_{1}, p_{2}\right) t\right), \gamma_{p_{1}, p_{2}}^{\prime}\left(\mathrm{d}_{M}\left(p_{1}, p_{2}\right) t\right)\right)
$$

where $p_{1}=\Phi^{-1}\left(y_{1}, 0\right)$ and $p_{2}=\Phi^{-1}\left(y_{2}, 1\right)$. 
Denote by $E$ the subset of $\mathbb{R}^{2}$ restricted by the curves $I \times\{0\}, I \times\{1\}$, $\Phi\left(\Gamma_{1}\right)$, and $\Phi\left(\Gamma_{2}\right)$. Given any $\left(x_{1}, x_{2}\right) \in E$, let

$$
\begin{aligned}
M_{x_{1}, x_{2}}=\left\{y_{1} \in I \mid\right. & \text { there is } y_{2} \in I \text { such that the geodesic } \\
& \left.\gamma_{\Phi^{-1}\left(y_{1}, 0\right), \Phi^{-1}\left(y_{2}, 1\right)} \text { goes through } \Phi^{-1}\left(x_{1}, x_{2}\right)\right\} .
\end{aligned}
$$

Note that for all $y_{1} \in M_{x_{1}, x_{2}}$ the point $y_{2} \in I$ in (3.3) is unique (provided $\left.x_{2}>0\right)$. Moreover, $M_{x_{1}, x_{2}} \neq \emptyset$ for all $\left(x_{1}, x_{2}\right) \in E$. For all $\left(x_{1}, x_{2}\right) \in E$, we define a function $F_{x_{1}, x_{2}}: M_{x_{1}, x_{2}} \rightarrow I$ by $F_{x_{1}, x_{2}}\left(y_{1}\right)=y_{2}$ where $y_{2}$ is as in (3.3). (If $x_{2}=0$ we obtain the vertical line segment $I$ above $y_{1}$.)

Lemma 3.1. The mapping $F_{x_{1}, x_{2}}$ has the following properties:

(1) If $\left(x_{1}, x_{2}\right),\left(\tilde{x}_{1}, x_{2}\right) \in E$ such that $\tilde{x}_{1}>x_{1}$, we have $F_{\tilde{x}_{1}, x_{2}}\left(y_{1}\right)>$ $F_{x_{1}, x_{2}}\left(y_{1}\right)$ for all $y_{1} \in M_{x_{1}, x_{2}} \cap M_{\tilde{x}_{1}, x_{2}}$.

(2) Given $\left(x_{1}, x_{2}\right),\left(\tilde{x}_{1}, x_{2}\right) \in E$ with $\tilde{x}_{1} \rightarrow x_{1}$, we have $F_{\tilde{x}_{1}, x_{2}}\left(y_{1}\right) \rightarrow$ $F_{x_{1}, x_{2}}\left(y_{1}\right)$ for all $y_{1} \in M_{x_{1}, x_{2}} \cap M_{\tilde{x}_{1}, x_{2}}$.

(3) For all $y_{1}, y_{2} \in I$ and $x_{2} \in I$ there exists $x_{1}$ such that $\left(x_{1}, x_{2}\right) \in$ $E$ and $F_{x_{1}, x_{2}}\left(y_{1}\right)=y_{2}$.

Proof. The claims follow directly from the definitions.

Given $t \in I$, let $L_{t}$ be the line in $\mathbb{R}^{2}$ which goes through $(1 / 2,1 / 2)$ and is orthogonal to the line segment going through the points in $\partial\left(I^{2}\right) \cap$ $\mathcal{G}\left(F_{1 / 2, t}\right)$. (Here the boundary of a set $A$ is denoted by $\partial A$ and the graph of a function $f$ by $\mathcal{G}(f)$.) Note that our assumptions guarantee that $\{(1 / 2, t) \mid t \in I\} \subset E$, and furthermore, the set $\partial\left(I^{2}\right) \cap \mathcal{G}\left(F_{1 / 2, t}\right)$ contains exactly two points.

We may choose $\widetilde{I}^{2} \subset I^{2}$ such that for all $t \in I$ and $\left(y_{1}, y_{2}\right) \in \widetilde{I}^{2}$ the intersection $L_{t} \cap \mathcal{G}\left(F_{x, t}\right)$ is a singleton for $x \in I$ with $F_{x, t}\left(y_{1}\right)=y_{2}$ (see Lemma $3.1(3))$. This enables us to define a function $P: I \times \widetilde{I}^{2} \rightarrow \mathbb{R}$ by

$$
P(t, y)=p_{t, y},
$$

where $y=\left(y_{1}, y_{2}\right) \in \widetilde{I}^{2}, p_{t, y}$ is the unique point in $L_{t} \cap \mathcal{G}\left(F_{x, t}\right)$, and the point $x$ is determined by $F_{x, t}\left(y_{1}\right)=y_{2}$. Here $L_{t}$ is identified with $\mathbb{R}$ such that the origin is at $(1 / 2,1 / 2)$.

Invariant measure under geodesic flow. Similarly as in [LL], we restrict our consideration to the normalized restriction measure $\widetilde{\mu}=$ $\left.\mu(\tilde{U})^{-1} \mu\right|_{\widetilde{U}}$, where $\widetilde{U}=\Psi\left(\widetilde{I}^{2} \times I\right)$. (Here $\left.\mu\right|_{\widetilde{U}}(A)=\mu(\widetilde{U} \cap A)$ for all $A \subset S M$.) Since $\mu$ is invariant under the geodesic flow, there is a measure $\nu$ on $\widetilde{I}^{2}$ such that $\Psi_{*}\left(\nu \times \mathcal{L}^{1}\right)=\widetilde{\mu}$.

Next we will present the measure $\Pi_{*} \widetilde{\mu}$ in a form which allows us to apply the general projection formalism of section 2. For this purpose, 
let $V=\Phi \circ \Pi(\widetilde{U})$. We define, for given $t \in I$ and $\omega \in(\Phi \circ \Pi \circ$ $\Psi)^{-1}\{(x, t) \mid(x, t) \in V\}$,

$$
B_{1}\left(\omega_{1}, \omega_{2}, \omega_{3}\right)=\left(\omega_{1}, \omega_{2}, t\right) .
$$

Clearly, $B_{1}: \widetilde{I}^{2} \times I \rightarrow \widetilde{I}^{2} \times I$ is a diffeomorphism. Setting $\widetilde{P}(\cdot, t)=$ $\left(P_{t}(\cdot), t\right)$, we find for all $(x, t) \in V$ a unique point $\tilde{x} \in \mathbb{R}$ such that $\widetilde{P} \circ B_{1}\left((\Phi \circ \Pi \circ \Psi)^{-1}\{(x, t)\}\right)=(\tilde{x}, t)$. Defining

$$
B_{2}(x, t)=(\tilde{x}, t)
$$

and using the fact that

$$
B_{1}\left((\Phi \circ \Pi \circ \Psi)^{-1}\{(x, t)\}\right)=\left\{\left(y_{1}, y_{2}, t\right) \mid y_{2}=F_{x, t}\left(y_{1}\right)\right\},
$$

we get a diffeomorphism $B_{2}: V \rightarrow B_{2}(V)$.

Lemma 3.2. The following properties hold:

(1) $(\Phi \circ \Pi)_{*} \widetilde{\mu}=\left(B_{2}^{-1} \circ \widetilde{P} \circ B_{1}\right)_{*}\left(\nu \times \mathcal{L}^{1}\right)$.

(2) For all non-negative Borel functions $f: \mathbb{R}^{2} \rightarrow \mathbb{R}$

$$
\int f(x, t) d\left(\widetilde{P}_{*}\left(\nu \times \mathcal{L}^{1}\right)\right)(x, t)=\iint f(x, t) d\left(\left(P_{t}\right)_{*} \nu\right)(x) d \mathcal{L}^{1}(t) .
$$

(3) For all non-negative Borel functions $g: \mathbb{R}^{3} \rightarrow \mathbb{R}$

$$
\int g d\left(\left(B_{1}\right)_{*}\left(\nu \times \mathcal{L}^{1}\right)\right)=\int g\left|\operatorname{det} D B_{1}^{-1}\right| d\left(\nu \times \mathcal{L}^{1}\right)
$$

where det $D B_{1}^{-1}$ is the determinant of the derivative of $B_{1}^{-1}$. Furthermore, there is a constant $C>0$ such that

$$
C^{-1} \leq\left|\operatorname{det} D B_{1}^{-1}\right| \leq C \text {. }
$$

(4) There exists a constant $C>0$ such that for all Borel sets $A \subset$ $\mathbb{R}^{2}$

$$
\frac{1}{C} \widetilde{P}_{*}\left(\nu \times \mathcal{L}^{1}\right)(A) \leq\left(\widetilde{P} \circ B_{1}\right)_{*}\left(\nu \times \mathcal{L}^{1}\right)(A) \leq C \widetilde{P}_{*}\left(\nu \times \mathcal{L}^{1}\right)(A) .
$$

(5) There is a constant $C>0$ such that

$$
C^{-1} \leq\left|\operatorname{det} D B_{2}^{-1}\right| \leq C \text {. }
$$

Proof. Clearly, (1) follows from the definitions, and (2) is a straightforward consequence of Fubini's theorem. Noting that $B_{1}$ can be written in the form $B_{1}\left(x_{1}, x_{2}, t\right)=\left(x_{1}, x_{2}, b\left(x_{1}, x_{2}, t\right)\right)$, Fubini's theorem gives the equality in (3). Our basic assumption (2) guarantees the existence of a constant $C$ such that $C^{-1} \leq\left|\operatorname{det} D\left(B_{1}^{-1}\right)\right| \leq C$ concluding the proof of (3). Finally, applying (3) gives (4), and (5) follows similarly as $(3)$. 


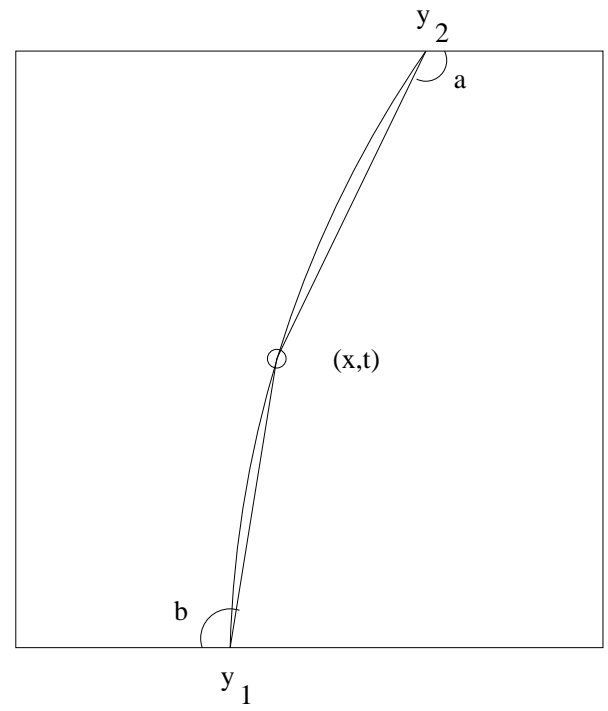

Figure 1. The notation for determining the slope of the graph $F_{x, t}$ at a point $\left(y_{1}, y_{2}\right)$

\section{Transversality ANd PRESERVAtion of HausdorfF DIMENSION IN 2-DIMENSIONAL MANIFOLDS}

In this section we discuss connections between [LL] and [PS]. In particular, we give a new proof of Theorem 1.1 which explains why the corresponding result fails if the dimension of the base manifold is more than 2 (see Remark 4.5). The machinery developed in this section leads us to prove in section 5 that the Radon-Nikodym derivative $\frac{d \Pi_{*} \mu}{d \mathcal{L}^{2}}$ has fractional derivatives in the Sobolev sense. An essential step is to prove that the function $T_{t}$, defined as in (2.2) in terms of the function $\mathrm{P}$ given in (3.4), has the crucial property of being transversal.

Proposition 4.1. Let $P$ be as in (3.4). Then (2.1) is satisfied. Furthermore, defining for all $t \in I$ and $x \neq y \in \widetilde{I}^{2}$

$$
T_{t}(x, y)=\frac{P(t, x)-P(t, y)}{|x-y|},
$$

properties (2.3) and (2.4) hold.

Proof. Observing that (2.1) and (2.4) follow directly from the definitions, it suffices to prove that the transversality condition (2.3) is satisfied.

Given $(x, t)$, let $\alpha$ be the slope of the graph of $F_{x, t}$ at a point $\left(y_{1}, y_{2}\right)$. Using the notation introduced in Figure 1, one may deduce the formula

$$
\tan \alpha=\frac{(1-t) \sin ^{2} b(x, t)}{t \sin ^{2} a(x, t)}
$$

from elementary geometrical arguments. Note that the basic assumption (2) in section 3 guarantees that both the angles $a$ and $b$ are 


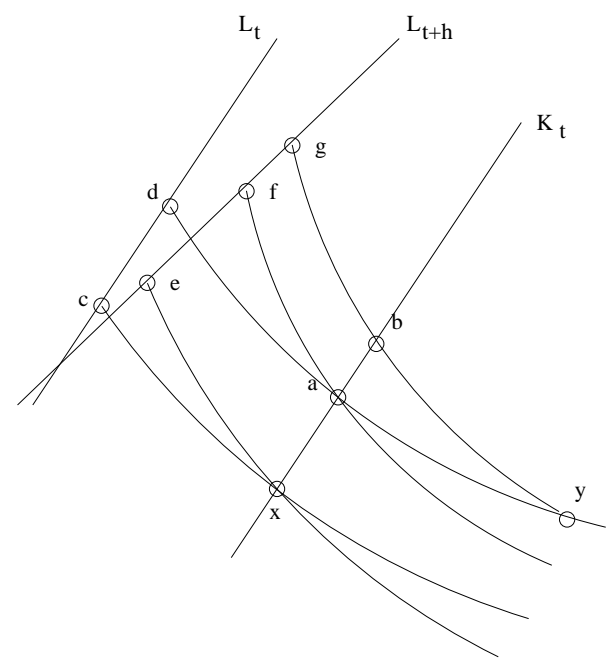

Figure 2. Above the line $K_{t}$ goes through $x$ and is parallel line to $L_{t},\{a\}=K_{t} \cap \mathcal{G}\left(F_{\cdot, t}\right),\{b\}=K_{t} \cap \mathcal{G}\left(F_{\cdot, t+h}\right)$, $c=P(t, x), d=P(t, y), e=P(t+h, x), f=P(t+h, a)$, and $g=P(t+h, y)$.

bounded away from 0 and $\pi$ and are close to each other. Combining this with equation (4.1), in turn, implies the existence of a positive constant $C_{1}$ such that

$$
\left|\frac{d \alpha}{d t}\right| \geq C_{1}
$$

for all $t$.

Letting $\varepsilon>0$, consider $x \neq y$ such that

$$
|P(t, x)-P(t, y)| \leq \varepsilon|x-y| \text {. }
$$

We will show that, choosing $\varepsilon$ small enough, we have for all small $h$

$$
|P(t+h, x)-P(t+h, y)-(P(t, x)-P(t, y))| \geq \varepsilon|x-y| h .
$$

This clearly gives transversality condition (2.3).

Note that our assumptions guarantee the existence of a constant $C_{F}$ (independent of $x, y$, and $t$ ) such that

$$
\begin{aligned}
& \max _{K \| L_{t}}\left\{\left|z_{1}-z_{2}\right| \mid z_{1} \in \mathcal{G}\left(F_{x, t}\right) \cap K, z_{2} \in \mathcal{G}\left(F_{y, t}\right) \cap K\right\} \\
& \leq C_{F} \min _{K \| L_{t}}\left\{\left|z_{1}-z_{2}\right| \mid z_{1} \in \mathcal{G}\left(F_{x, t}\right) \cap K, z_{2} \in \mathcal{G}\left(F_{y, t}\right) \cap K\right\},
\end{aligned}
$$

where both the maximum and the minimum are taken over all lines $K$ that are parallel to $L_{t}$ (denoted by the symbol $K \| L_{t}$ ). Using the notation shown in Figure 2, we have

$$
\begin{aligned}
& |x-a| \leq \varepsilon C_{F}|x-y| \\
& |a-b| \geq C_{2}|x-y| h \\
& || c-d|-| e-f|| \leq C_{3} \varepsilon|x-y| h,
\end{aligned}
$$




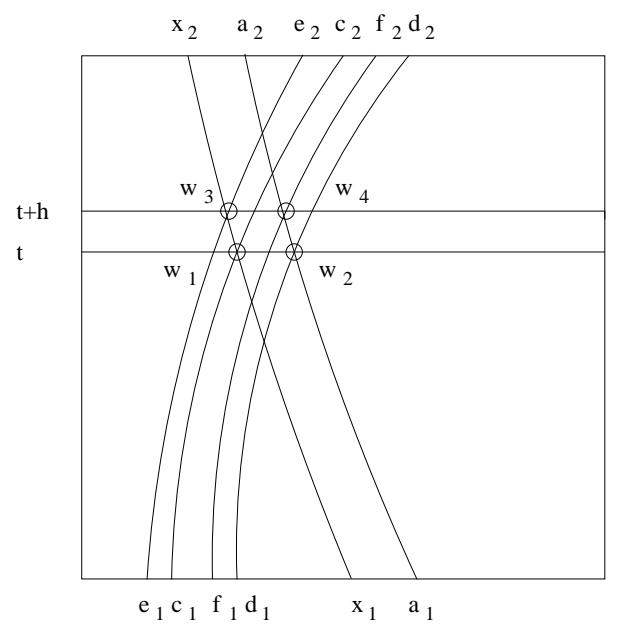

Figure 3. The setting for the proof of the last inequality in (4.6). The notation corresponds to Figure 2 in a natural way.

where both $C_{2}$ and $C_{3}$ are constants that do not depend on $x, y$, and $t$. In fact, the first inequality in (4.6) is a consequence of (4.5) and (4.3). Choosing $\varepsilon<1 /\left(2 C_{F}\right)$, the second inequality follows from the first one and the fact that there is a constant $C$ such that $|a-b| \geq C|a-y| h$ (see (4.2)). For the last one, observe first that, since the geodesics are close to lines in $V$ and depend smoothly on the initial data, there is a constant $C$ (independent of $x, y$, and $t$ ) such that

$$
|| c-d|-| e-f|| \leq C|| w_{1}-w_{2}|-| w_{3}-w_{4}||
$$

where $w_{1}, w_{2}, w_{3}$, and $w_{4}$ are as in Figure 3. Using the fact that the closer to each other the geodesics are, the more they look like parallel curves in $V$, we get

$$
|| w_{1}-w_{2}|-| w_{3}-w_{4}|| \leq \widetilde{C}\left|w_{1}-w_{2}\right| h \leq \widehat{C}|x-a| h .
$$

(Here $\widetilde{C}$ and $\widehat{C}$ are constants that are independent of $x, y$, and $t$.) This, in turn, combined with (4.7) and the first inequality in (4.6), completes the proof of the last inequality of (4.6).

Finally, after noting that for small $h$ we have $|f-g| \geq\left(1 /\left(2 C_{F}\right)\right)|a-b|$ by (4.5), we deduce from (4.6)

$$
|| c-d|-| e-g||=|f-g|-|| c-d|-| e-f|| \geq C_{3} \varepsilon|x-y| h
$$

for $\varepsilon<\min \left\{1 /\left(2 C_{F}\right), C_{2} /\left(4 C_{F} C_{3}\right)\right\}$. Hence (4.4) follows.

As a corollary of Proposition 4.1, one obtains quite easily a new proof for Theorem 1.1. This is achieved by means of Proposition 4.2. Recall that the Hausdorff dimension of a finite Borel measure $\mu$ on a Riemannian manifold $X$ is defined by means of local lower dimensions, 
$\underline{\operatorname{dim}}_{\mathrm{loc}}$, as follows:

$$
\operatorname{dim}_{\mathrm{H}} \mu=\mu \text { - } \underset{x \in X}{\operatorname{ess}} \inf _{\underline{\operatorname{dim}}} \mu(x)
$$

where

$$
\underline{\operatorname{dim}}_{\operatorname{loc}} \mu(x)=\liminf _{r \rightarrow 0} \frac{\log \mu(B(x, r))}{\log r} .
$$

Here $B(x, r)$ is the open ball with centre at $x$ and radius $r>0$. The following equality relates Hausdorff dimension of measures to that of sets:

$$
\operatorname{dim}_{\mathrm{H}} \mu=\inf \left\{\operatorname{dim}_{\mathrm{H}} A \mid A \text { is a Borel set with } \mu(A)>0\right\} .
$$

Proposition 4.2. With the notation introduced in section 3, we have:

(1) Assuming that $\operatorname{dim}_{\mathrm{H}} \nu \leq 1$, we have $\operatorname{dim}_{\mathrm{H}}\left(P_{t}\right)_{*} \nu=\operatorname{dim}_{\mathrm{H}} \nu$ for $\mathcal{L}^{1}$-almost all $t \in(0,1)$.

(2) Assuming that $\operatorname{dim}_{\mathrm{H}} \nu>1$, we have $\left(P_{t}\right)_{*} \nu \ll \mathcal{L}^{1}$ for $\mathcal{L}^{1}$-almost all $t \in(0,1)$.

Proof. To verify (1), let $\beta<\operatorname{dim}_{\mathrm{H}} \nu$. Defining $\nu_{i}=\left.\nu\right|_{A_{i}}$ for all $i=$ $1,2, \ldots$, where

$$
A_{i}=\left\{x \in \mathbb{R}^{2} \mid \nu(B(x, r)) \leq i r^{\beta} \text { for all } r>0\right\},
$$

on easily checks that $I_{\alpha}\left(\nu_{i}\right)<\infty$ for all $\alpha<\beta$, and $\nu_{i}(A) \rightarrow \nu(A)$ for all $A \subset \mathbb{R}^{2}$. Given $\sigma<\alpha$, we get from inequality (2.6) in Theorem 2.1 that for $\mathcal{L}^{1}$-almost all $t \in(0,1)$

$$
\operatorname{dim}_{\mathrm{H}}\left(\left(P_{t}\right)_{*} \nu_{i}\right) \geq \operatorname{dim}_{\mathrm{S}}\left(\left(P_{t}\right)_{*} \nu_{i}\right)>\sigma
$$

for all $i$. This, in turn, implies that $\operatorname{dim}_{\mathrm{H}}\left(\left(P_{t}\right)_{*} \nu\right) \geq \sigma$ for $\mathcal{L}^{1}$-almost all $t \in(0,1)$. Finally, taking a sequence $\sigma_{j} \rightarrow \operatorname{dim}_{\mathrm{H}} \nu$, gives (1), since $P_{t}$ does not increase dimension as a Lipschitz function.

For (2), we consider $1<\beta<\operatorname{dim}_{\mathrm{H}} \nu$ and proceed as above to find a sequence $\left(\nu_{i}\right)$ of measures with $I_{\beta}\left(\nu_{i}\right)<\infty$ such that $\nu_{i}(A) \rightarrow \nu(A)$ for all $A \subset \mathbb{R}^{2}$. Now inequality (2.5) in Theorem 2.1 implies that for $\mathcal{L}^{1}$-almost all $t \in(0,1)$ one has $\left(\left(P_{t}\right)_{*} \nu_{i}\right)^{\wedge} \in L^{2}$ for all $i$, and therefore $\left(P_{t}\right)_{*} \nu_{i} \ll \mathcal{L}^{1}$ for all $i$. This gives $(2)$.

We continue by explaining how Theorem 1.1 follows from Proposition 4.2. For this purpose we need two intermediate steps:

Corollary 4.3. Using the same notation as in section 3, we have:

(1) If $\operatorname{dim}_{\mathrm{H}} \widetilde{\mu} \leq 2$, then $\operatorname{dim}_{\mathrm{H}}\left(\widetilde{P}_{*}\left(\nu \times \mathcal{L}^{1}\right)\right)=\operatorname{dim}_{\mathrm{H}} \widetilde{\mu}$.

(2) If $\operatorname{dim}_{\mathrm{H}} \widetilde{\mu}>2$, then $\widetilde{P}_{*}\left(\nu \times \mathcal{L}^{1}\right) \ll \mathcal{L}^{2}$.

Proof. Note that $\operatorname{dim}_{\mathrm{H}} \widetilde{\mu}=\operatorname{dim}_{\mathrm{H}} \nu+1$ (see $[\mathrm{H}]$ ). To prove (1), Proposition $4.2(1)$ gives $\operatorname{dim}_{\mathrm{H}}\left(P_{t}\right)_{*} \nu=\operatorname{dim}_{\mathrm{H}} \nu$ for $\mathcal{L}^{1}$-almost all $t \in \mathbb{R}$. From Lemma 2.2 and Lemma $3.2(2)$, we deduce that $\operatorname{dim}_{\mathrm{H}}\left(\widetilde{P}_{*}\left(\nu \times \mathcal{L}^{1}\right)\right) \geq$ $\operatorname{dim}_{\mathrm{H}} \nu+1=\operatorname{dim}_{\mathrm{H}} \widetilde{\mu}$. The fact that $\widetilde{P}$ is a Lipschitz mapping yields to $(1)$. 
For (2), let $A \subset \mathbb{R}^{2}$ be a Borel set with $\mathcal{L}^{2}(A)=0$. Setting $A_{t}=$ $\{x \in \mathbb{R} \mid(x, t) \in A\}$ for all $t \in \mathbb{R}$, and using Fubini's theorem and Proposition $4.2(2)$, we get $\left(P_{t}\right)_{*} \nu\left(A_{t}\right)=0$ for $\mathcal{L}^{1}$-almost all $t \in \mathbb{R}$. Combining this with Lemma 3.2 (2) concludes the proof.

Corollary 4.4. Using the notation given in section 3, we have:

(1) If $\operatorname{dim}_{\mathrm{H}} \widetilde{\mu} \leq 2$, then $\operatorname{dim}_{\mathrm{H}}(\Phi \circ \Pi)_{*} \widetilde{\mu}=\operatorname{dim}_{\mathrm{H}} \widetilde{\mu}$.

(2) If $\operatorname{dim}_{\mathrm{H}} \widetilde{\mu}>2$, then $(\Phi \circ \Pi)_{*} \widetilde{\mu} \ll \mathcal{L}^{2}$.

Proof. Corollary 4.3, Lemma 3.2 (4), and the fact that $B_{2}^{-1}$ is a biLipschitz mapping (see Lemma $3.2(5))$ combine to give that $\operatorname{dim}_{\mathrm{H}}\left(B_{2}^{-1}\right.$ o $\left.\widetilde{P} \circ B_{1}\right)_{*}\left(\nu \times \mathcal{L}^{1}\right)=\operatorname{dim}_{\mathrm{H}} \widetilde{\mu}$ provided that $\operatorname{dim}_{\mathrm{H}} \widetilde{\mu} \leq 2$, and furthermore, $\left(B_{2}^{-1} \circ \widetilde{P} \circ B_{1}\right)_{*}\left(\nu \times \mathcal{L}^{1}\right) \ll \mathcal{L}^{2}$ under the assumption $\operatorname{dim}_{\mathrm{H}} \widetilde{\mu}>2$. This in turn gives the claim by Lemma $3.2(1)$.

Since $\Phi$ is bi-Lipschitz mapping, Theorem 1.1 follows immediately from Corollary 4.4 by representing the original measure $\mu$ as a finite sum of measures $\widetilde{\mu}_{i}$ having the same properties as the measure $\widetilde{\mu}$ above.

Remark 4.5. In section 6 we construct examples which show that Theorem 1.1 fails for higher dimensional base manifolds. The reason for the failure, which may be deduced from the above methods, is as follows: The local invariance produces parametrized family of projections onto $(n-1)$-dimensional planes in $2(n-1)$-dimensional space. The parameter is given by the time coordinate, and therefore the family is 1-dimensional. Since the dimension of the space of $(n-1)$-planes in $2(n-1)$ dimensional space is greater than 1 , if $n \geq 3$, the transversality condition cannot hold.

\section{Fractional DeRivatives}

In this section we answer to the question concerning the fractional derivatives of the density of the projected measure $\Pi_{*} \mu$ addressed in [LL]. The main theorem of this section is as follows:

Theorem 5.1. Let $M$ be a compact Riemannian surface and let $\Pi$ : $S M \rightarrow M$ be the natural projection from the unit tangent bundle $S M$ onto the base manifold $M$. Assume that $\mu$ is a Radon probability measure on $S M$ such that $\mu$ is invariant under the geodesic flow and $I_{\alpha}(\mu)<\infty$ for some $\alpha>2$. Then for all $\gamma<(\alpha-2) / 2$ the projected measure $\Pi_{*} \mu$ has fractional derivatives of order $\gamma$ in $L^{2}$, that is, $\left\|\Pi_{*} \mu\right\|_{2, \gamma}<\infty$.

Below the proof of Theorem 5.1 is divided into a sequence of lemmas. Observe that Theorem 2.1 combined with Proposition 4.1 implies the existence of fractional derivatives for almost all horizontal slices of $\Pi_{*} \mu$, which are, in fact, diffeomorphic images of the measures $\left(P_{t}\right)_{*} \nu$. However, since this approach does not give the desired result for the measure $\Pi_{*} \mu$, we modify the methods of $[\mathrm{PS}]$ in a more effective way. 
Using the same notation as in the previous sections, we begin with a small technical lemma.

Lemma 5.2. Let $\alpha>1$. Assume that $\mu=F_{*}\left(\nu \times\left.\mathcal{L}^{1}\right|_{K}\right)$, where $K \subset \mathbb{R}$ is a compact set and $F$ is a diffeomorphism such that $C^{-1} \leq$ $|\operatorname{det} D F| \leq C$ for some $C>0$. Then

$$
I_{\alpha}(\mu)<\infty \Longleftrightarrow I_{\alpha-1}(\nu)<\infty \text {. }
$$

Proof. The claim follows from straightforward calculations.

Next lemma shows that for fixed $q \neq q^{\prime} \in \widetilde{I}^{2}$ the mapping $a \mapsto$ $T_{a}\left(q, q^{\prime}\right)$ is small only in neighbourhoods of finitely many zeroes.

Lemma 5.3. For any $q \neq q^{\prime} \in \widetilde{I}^{2}$ there exist $a_{1}, \ldots, a_{N} \in I$ such that

$$
\left\{a \in I|| T_{a}\left(q, q^{\prime}\right) \mid \leq d\right\} \subset \bigcup_{i=1}^{N} B\left(a_{i}, C_{T}^{-1} d\right)
$$

for all $d<C_{T}$. Moreover, the mapping $a \mapsto T_{a}\left(q, q^{\prime}\right)$ is a diffeomorphism on $B\left(a_{i}, C_{1}^{-1} C_{T}\right)$ for all $i=1, \ldots, N$, and $N \leq C_{1} / C_{T}+2$. (Here $C_{T}$ is as in (2.3) and $C_{1}$ as in (2.4).)

Proof. Let $a_{1}, \ldots, a_{N-2}$ be the zeroes of the function $a \mapsto T_{a}\left(q, q^{\prime}\right)$, and let $a_{N-1}=0$ and $a_{N}=1$. Then all the claims follow from (2.3) and $(2.4)$.

We continue by defining mappings $F_{q, q^{\prime}}$ and by studying their basic properties which will be needed in the proof of Lemma 5.6.

Lemma 5.4. Given $q \neq q^{\prime} \in \widetilde{I}^{2}$, let $r=\left|q-q^{\prime}\right|$. Define $F_{q, q^{\prime}}: I^{2} \rightarrow \mathbb{R}^{2}$ by

$$
F_{q, q^{\prime}}(a, b)=\left(T_{a}\left(q, q^{\prime}\right)+r^{-1}\left(P_{a}\left(q^{\prime}\right)-P_{b}\left(q^{\prime}\right)\right), r^{-1}(a-b)\right) .
$$

Let $a_{1}, \ldots, a_{N} \in I$ be as in Lemma 5.3. For any $i=1, \ldots, N$, set

$$
\begin{array}{r}
O_{i}=\left\{(a, b) \in\left(B\left(a_{i}, C_{1}^{-1} C_{T}\right) \cap(0,1)\right) \times(0,1)|| T_{a}\left(q, q^{\prime}\right) \mid<C_{T}\right. \text { and } \\
\left.|a-b|<\left(2 \widetilde{C}_{2}\right)^{-1} C_{T} r\right\},
\end{array}
$$

where $\widetilde{C}_{2}, C_{T}$, and $C_{1}$ are as in (2.1), (2.3), and (2.4), respectively. Then the restriction of $F_{q, q^{\prime}}$ to the set $O_{i}$ is a diffeomorphism onto $F_{q, q^{\prime}}\left(O_{i}\right)$. Furthermore, there are constants $c$ and $c(l)$ for all $l \in \mathbb{N}$ which are independent of $q$ and $q^{\prime}$ such that

$$
\| D F_{q, q^{\prime}}^{-1}||<c,\left|\partial^{\eta} F_{q, q^{\prime}}^{-1}\right|<c(|\eta|) \text {, and }\left|\partial^{\eta} \operatorname{det} D F_{q, q^{\prime}}^{-1}\right|<c(|\eta|)
$$

for all indices $\eta=\left(\eta_{1}, \eta_{2}\right) \in \mathbb{N}^{2}$. Here $|\eta|=\eta_{1}+\eta_{2}$ and $\partial^{\eta}=\partial_{a}^{\eta_{1}} \partial_{b}^{\eta_{2}}$.

Proof. By (2.1) and (2.3) we have for all $(a, b) \in O_{i}$

$$
\begin{aligned}
\left|\operatorname{det} D F_{q, q^{\prime}}(a, b)\right| & =r^{-1}\left|\partial_{a} T_{a}\left(q, q^{\prime}\right)-r^{-1}\left(\partial_{b} P_{b}\left(q^{\prime}\right)-\partial_{a} P_{a}\left(q^{\prime}\right)\right)\right| \\
& \geq(2 r)^{-1} C_{T} .
\end{aligned}
$$


For the the first claim it is therefore sufficient to show that the restriction of $F_{q, q^{\prime}}$ to $O_{i}$ is an injection. This, in turn, follows from two easy observations: If $(a, b),\left(a^{\prime}, b^{\prime}\right) \in O_{i}$ with $a-b \neq a^{\prime}-b^{\prime}$, then clearly $F_{q, q^{\prime}}(a, b) \neq F_{q, q^{\prime}}\left(a^{\prime}, b^{\prime}\right)$. On the other hand, $F_{q, q^{\prime}}$ is strictly monotone on the line segments $\left\{(a, b) \in O_{i} \mid b-a=d\right\}$, where $d \in \mathbb{R}$, since

$$
\left|\partial_{a} T_{a}\left(q, q^{\prime}\right)-r^{-1}\left(\partial_{a} P_{a+d}\left(q^{\prime}\right)-\partial_{a} P_{a}\left(q^{\prime}\right)\right)\right| \geq 2^{-1} C_{T} .
$$

For (5.1) note that

$$
\begin{aligned}
D F_{q, q^{\prime}}^{-1}(y) & =\left(\operatorname{det} D F_{q, q^{\prime}}\left(F_{q, q^{\prime}}^{-1}(y)\right)\right)^{-1}\left(\begin{array}{cc}
-r^{-1} & r^{-1} \partial_{b} P_{b}\left(q^{\prime}\right) \\
-r^{-1} & \partial_{a} T_{a}\left(q, q^{\prime}\right)+r^{-1} \partial_{a} P_{a}\left(q^{\prime}\right)
\end{array}\right) \\
& =:\left(\operatorname{det} D F_{q, q^{\prime}}\left(F_{q, q^{\prime}}^{-1}(y)\right)\right)^{-1} A,
\end{aligned}
$$

where $(a, b)=F_{q, q^{\prime}}^{-1}(y)$. Combining this with inequality (5.2), (2.1), and (2.4), gives $\left\|D F_{q, q^{\prime}}^{-1}\right\|<c$. Using similar arguments and the fact that for all $l \in \mathbb{N}$ there exists a constant $C(l)$ such that $\left|\partial^{\eta} A_{i j}\right|<r^{-1} C(|\eta|)$ for all $\eta$ and $i, j$, the second claim in (5.1) follows by induction. Finally, the last estimate is a consequence of the previous one.

In the following lemma which is from [PS] we denote by $\mathcal{S}\left(\mathbb{R}^{n}\right)$ the Schwartz space of smooth functions such that all of their derivatives decay faster than any power.

Lemma 5.5. There exists $\psi \in \mathcal{S}\left(\mathbb{R}^{n}\right)$ such that $\hat{\psi}>0$, spt $\hat{\psi} \subset\{\xi \in$ $\left.\mathbb{R}^{n}|1 \leq| \xi \mid \leq 4\right\}$, and $\sum_{j=-\infty}^{\infty} \hat{\psi}\left(2^{-j} \xi\right)=1$ for all $\xi \neq 0$. Furthermore, for any finite Radon measure $\nu$ on $\mathbb{R}^{n}$ and any $\gamma \in \mathbb{R}$ there exists a constant $C$ such that

$$
\frac{1}{C}\|\nu\|_{2, \gamma}^{2} \leq \sum_{j=-\infty}^{\infty} 2^{2 j \gamma} \int_{\mathbb{R}^{n}}\left(\psi_{2^{-j}} * \nu\right)(x) d \nu(x) \leq C\|\nu\|_{2, \gamma}^{2},
$$

where $\psi_{2^{-j}}(x)=2^{j n} \psi\left(2^{j} x\right)$. (Above $*$ is the convolution.)

Proof. See [PS, Lemma 4.1].

Next we prove a lemma which is a modification of [PS, Lemma 7.10] tailored for our purposes.

Lemma 5.6. Assume that $\rho$ is a smooth non-negative real valued function which is supported inside the open unit square $(0,1)^{2}$. Let $\psi$ be as in Lemma 5.5. Then for all $q, q^{\prime} \in \widetilde{I}^{2}$ with $q \neq q^{\prime}, j \in \mathbb{Z}$, and $k \in \mathbb{N} \backslash\{0\}$ we have

$$
\begin{gathered}
\left|\int_{\mathbb{R}} \int_{\mathbb{R}} \rho(a, b) \psi\left(2^{j}\left(P_{a}(q)-P_{b}\left(q^{\prime}\right), a-b\right)\right) d \mathcal{L}^{1}(a) d \mathcal{L}^{1}(b)\right| \\
\leq C \min \left\{\left(1+2^{j}\left|q-q^{\prime}\right|\right)^{-k},\left(1+2^{j}\right)^{-1}\right\},
\end{gathered}
$$

where the constant $C$ does not depend on $q, q^{\prime}$, and $j$. 
Proof. Observing that it is enough to study positive integers $j$, and using the fast decay of $\psi$ we have

$$
\begin{aligned}
& \left|\int_{\mathbb{R}} \rho(a, b) \psi\left(2^{j}\left(P_{a}(q)-P_{b}\left(q^{\prime}\right)\right), 2^{j}(a-b)\right) d \mathcal{L}^{1}(a)\right| \\
& \quad \leq c 2^{-j}+c^{\prime} \int_{t>2^{-j}}\left(2^{j} t\right)^{-2} d \mathcal{L}^{1}(t) \leq C\left(1+2^{j}\right)^{-1} .
\end{aligned}
$$

For the other upper bound, fix $k, j \in \mathbb{N}$ such that $k \geq 1$. Setting $r=\left|q-q^{\prime}\right|$, we may assume that $2^{j} r>1$. Let $\phi: \mathbb{R}^{2} \rightarrow \mathbb{R}$ be a smooth function such that $0 \leq \phi \leq 1, \phi \equiv 1$ on $[-1,1]^{2}$, and $\phi \equiv 0$ on $\mathbb{R}^{2} \backslash[-2,2]^{2}$. Letting $F_{q, q^{\prime}}: I^{2} \rightarrow \mathbb{R}^{2}$ be as in Lemma 5.4 , one obtains

$$
\begin{aligned}
& \int_{\mathbb{R}} \int_{\mathbb{R}} \rho(a, b) \psi\left(2^{j}\left(P_{a}(q)-P_{b}\left(q^{\prime}\right), a-b\right)\right) d \mathcal{L}^{1}(a) d \mathcal{L}^{1}(b) \\
& =\int_{\mathbb{R}} \int_{\mathbb{R}} \rho(a, b) \psi\left(2^{j} r F_{q, q^{\prime}}(a, b)\right) \phi\left(C_{T}^{-1} F_{q, q^{\prime}}(a, b)\right) d \mathcal{L}^{1}(a) d \mathcal{L}^{1}(b) \\
& \quad+\int_{\mathbb{R}} \int_{\mathbb{R}} \rho(a, b) \psi\left(2^{j} r F_{q, q^{\prime}}(a, b)\right)\left(1-\phi\left(C_{T}^{-1} F_{q, q^{\prime}}(a, b)\right)\right) d \mathcal{L}^{1}(a) d \mathcal{L}^{1}(b) \\
& =: A_{1}+A_{2} .
\end{aligned}
$$

Since the integrand of $A_{2}$ is non-zero only if $\left|F_{q, q^{\prime}}\right|>C_{T}$, the fact that the support spt $\rho$ of $\rho$ is inside $(0,1)^{2}$ and $\psi \in \mathcal{S}\left(\mathbb{R}^{2}\right)$ implies

$$
\left|A_{2}\right| \leq \int_{\mathbb{R}} \int_{\mathbb{R}} \rho(a, b)\left(C_{T} 2^{j} r\right)^{-k} d \mathcal{L}^{1}(a) d \mathcal{L}^{1}(b) \leq C\left(1+2^{j} r\right)^{-k} .
$$

We continue by estimating $A_{1}$. Picking $a_{1}, \ldots, a_{N}$ as in Lemma 5.3, we find $d_{2}, d_{3}<\min \left\{C_{T}, C_{1}^{-1} C_{T}\right\}$ such that

$$
\left\{a \in(0,1)|| T_{a}\left(q, q^{\prime}\right) \mid \leq d_{3}\right\} \subset \bigcup_{i=1}^{N} B\left(a_{i}, d_{2} / 2\right)
$$

and

$$
\bigcup_{i=1}^{N} B\left(a_{i}, d_{2}\right) \cap(0,1) \subset\left\{a \in(0,1)|| T_{a}\left(q, q^{\prime}\right) \mid \leq C_{T} / 4\right\} .
$$

Let $d_{1}<\min \left\{\left(2 \widetilde{C}_{2}\right)^{-1} C_{T},\left(4 \widetilde{C}_{1}\right)^{-1} C_{T}\right\}$. For all $i=0, \ldots, N$, there exists a smooth function $\chi_{i}: \mathbb{R} \rightarrow[0,1]$ with the following properties:

(1) spt $\chi_{0} \subset B\left(0, d_{1}\right)$.

(2) spt $\chi_{i} \subset B\left(a_{i}, d_{2}\right)$ for all $i=1, \ldots, N$.

(3) Letting $O_{i}$ be as in Lemma 5.4, we have

$$
\chi_{0}\left(r^{-1}(a-b)\right) \chi_{i}(a)=0
$$

for all $i=1, \ldots, N$ and $(a, b) \in(0,1)^{2} \backslash O_{i}$. 
(4) For all $(a, b) \in \operatorname{spt} \rho$ with $\left|T_{a}\left(q, q^{\prime}\right)\right| \leq d_{3}$ and $r^{-1}|a-b| \leq$ $\left(8 \widetilde{C}_{1}\right)^{-1} d_{3}$ we have

$$
\sum_{i=1}^{N} \chi_{0}\left(r^{-1}(a-b)\right) \chi_{i}(a)=1 .
$$

(5) For all $l \in N$ there is a constant $c_{l}$ such that

$$
\sup _{0 \leq i \leq N}\left\|\partial^{l} \chi_{i}\right\|_{\infty} \leq c_{l}
$$

(Note that above property (3) follows from (1), (2), and (5.4), and (5.3) makes the choice of property (4) possible.) Combining (2.1), (5.4), and properties (2) and (3) leads to

$$
\chi_{0}\left(r^{-1}(a-b)\right) \chi_{i}(a)=\chi_{0}\left(r^{-1}(a-b)\right) \chi_{i}(a) \phi\left(C_{T}^{-1} F_{q, q^{\prime}}(a, b)\right)
$$

for all $(a, b) \in \mathbb{R}^{2}$ and $i=1, \ldots, N$. (This follows from the fact that $\phi\left(C_{T}^{-1} F_{q, q^{\prime}}(a, b)\right)=1$ if the left hand side in the above equality is non zero.) Therefore

$$
\begin{gathered}
A_{1}=\sum_{i=1}^{N} \int_{\mathbb{R}} \int_{\mathbb{R}} \rho(a, b) \chi_{0}\left(r^{-1}(a-b)\right) \chi_{i}(a) \psi\left(2^{j} r F_{q, q^{\prime}}(a, b)\right) d \mathcal{L}^{1}(a) d \mathcal{L}^{1}(b) \\
+\int_{\mathbb{R}} \int_{\mathbb{R}} \rho(a, b)\left(1-\sum_{i=1}^{N} \chi_{0}\left(r^{-1}(a-b)\right) \chi_{i}(a)\right) \psi\left(2^{j} r F_{q, q^{\prime}}(a, b)\right) \\
\times \phi\left(C_{T}^{-1} F_{q, q^{\prime}}(a, b)\right) d \mathcal{L}^{1}(a) d \mathcal{L}^{1}(b)=: \sum_{i=1}^{N} D_{i}+D .
\end{gathered}
$$

From (4) we deduce that on the support of the integrand of $D$ we have $\left|F_{q, q^{\prime}}(a, b)\right| \geq\left(8 \widetilde{C}_{1}\right)^{-1} d_{3}$, and so, similarly as before, we get

$$
|D| \leq C\left(1+2^{j} r\right)^{-k}
$$

Since $N \leq C_{1} / C_{T}+2$ by Lemma 5.3 , it suffices to show that each $D_{i}$ has an upper bound of the desired form. Fixing $1 \leq i \leq N$ and applying (3) and Lemma 5.4 gives

$$
\begin{array}{r}
D_{i}=\int_{O_{i}} \rho(a, b) \chi_{0}\left(r^{-1}(a-b)\right) \chi_{i}(a) \psi\left(2^{j} r F_{q, q^{\prime}}(a, b)\right) d \mathcal{L}^{2}(a, b) \\
=\int_{F_{q, q^{\prime}}\left(O_{i}\right)} \rho\left(F_{q, q^{\prime}}^{-1}(u, v)\right) \chi_{0}(v) \chi_{i}\left(\left(F_{q, q^{\prime}}^{-1}\right)_{1}(u, v)\right) \psi\left(2^{j} r(u, v)\right) \\
\times\left|\operatorname{det}\left(D F_{q, q^{\prime}}^{-1}(u, v)\right)\right| d \mathcal{L}^{2}(u, v),
\end{array}
$$

where $\left(F_{q, q^{\prime}}^{-1}\right)_{1}(u, v)$ is the first coordinate of $F_{q, q^{\prime}}^{-1}(u, v)$. Since the integrand of $D_{i}$ is zero outside $O_{i}$ by (3), we may modify $F_{q, q^{\prime}}$ in such a way that it becomes a diffeomorphism on $\mathbb{R}^{2}$, and all the bounds given in Lemma 5.4 remain unchanged. Defining for all $(u, v) \in \mathbb{R}^{2}$

$$
G(u, v):=\rho\left(F_{q, q^{\prime}}^{-1}(u, v)\right) \chi_{0}(v) \chi_{i}\left(\left(F_{q, q^{\prime}}^{-1}\right)_{1}(u, v)\right)\left|\operatorname{det}\left(D F_{q, q^{\prime}}^{-1}(u, v)\right)\right|,
$$


and choosing $0<\varepsilon<1$ such that $(k+2)(1-\varepsilon)>k$, we rewrite $D_{i}$ as

$$
\begin{aligned}
D_{i} & =\int_{|y|<\left(2^{j} r\right)^{\varepsilon-1}} G(y) \psi\left(2^{j} r y\right) d \mathcal{L}^{2}(y)+\int_{|y|>\left(2^{j} r\right)^{\varepsilon-1}} G(y) \psi\left(2^{j} r y\right) d \mathcal{L}^{2}(y) \\
& =: J_{1}+J_{2} .
\end{aligned}
$$

From (5.1) we obtain that

$$
\left|J_{2}\right| \leq c \int_{t>\left(2^{j} r\right)^{\varepsilon-1}}\left(2^{j} r t\right)^{-\frac{k}{\varepsilon}-1} t d \mathcal{L}^{1}(t) \leq C\left(1+2^{j} r\right)^{-k-1},
$$

and therefore, it remains to estimate $J_{1}$.

Note that $\psi$ has vanishing moments of all orders since $\partial^{\eta} \hat{\psi}(0)=0$ for all $\eta$. Using Taylor expansion for the function $G$, we calculate

$$
\begin{aligned}
J_{1}= & -\sum_{|\eta|<k} \int_{|y|>\left(2^{j} r\right)^{\varepsilon-1}}(\eta !)^{-1} \partial^{\eta} G(0) y^{\eta} \psi\left(2^{j} r y\right) d \mathcal{L}^{2}(y) \\
& +\sum_{|\eta|=k} \int_{|y|<\left(2^{j} r\right)^{\varepsilon-1}}(\eta !)^{-1} \partial^{\eta} G(t(y) y) \psi\left(2^{j} r y\right) y^{\eta} d \mathcal{L}^{2}(y) \\
= & :-\sum_{|\eta|<k} K_{\eta}+K .
\end{aligned}
$$

Here $y^{\eta}=y_{1}^{\eta_{1}} y_{2}^{\eta_{2}}, \eta !=\eta_{1} ! \eta_{2} !$, and $t(y) \in[0,1]$. Finally,

$$
\begin{aligned}
|K| & \leq c \int_{|y|<\left(2^{j} r\right)^{\varepsilon-1}} \sup _{|\eta|=k}\left\|\partial^{\eta} G\right\|_{\infty}|y|^{k} d \mathcal{L}^{2}(y) \\
& \leq c \sup _{|\eta|=k}\left\|\partial^{\eta} G\right\|_{\infty}\left(2^{j} r\right)^{-(1-\varepsilon)(k+2)} \leq C \sup _{|\eta|=k}\left\|\partial^{\eta} G\right\|_{\infty}\left(1+2^{j} r\right)^{-k}
\end{aligned}
$$

and

$$
\begin{aligned}
\left|K_{\eta}\right| & \leq c\left\|\partial^{\eta} G\right\|_{\infty} \int_{|y|>\left(2^{j} r\right)^{\varepsilon-1}}|y|^{|\eta|}\left|2^{j} r y\right|^{-|\eta|-1-\frac{k}{\varepsilon}} d \mathcal{L}^{2}(y) \\
& \leq C\left\|\partial^{\eta} G\right\|_{\infty}\left(1+2^{j} r\right)^{-k-1} .
\end{aligned}
$$

Thus the claim follows from Lemma 5.4.

As an immediate consequence of Lemma 5.6 we obtain the following result.

Corollary 5.7. Let $\rho$ and $\psi$ be as in Lemma 5.6, and let $q, q^{\prime} \in \widetilde{I}^{2}$ with $q \neq q^{\prime}$. Then for any $k, n \in \mathbb{N} \backslash\{0\}$ we have

$$
\begin{gathered}
\left|\int_{\mathbb{R}} \int_{\mathbb{R}} \rho(a, b) \psi\left(2^{j}\left(P_{a}(q)-P_{b}\left(q^{\prime}\right), a-b\right)\right) d \mathcal{L}^{1}(a) d \mathcal{L}^{1}(b)\right| \\
\leq C\left(1+2^{j}\left|q-q^{\prime}\right|\right)^{-\frac{k}{n}}\left(1+2^{j}\right)^{-\frac{n-1}{n}}
\end{gathered}
$$

where $C$ does not depend on $q, q^{\prime}$ or $j$. 
Proof of Theorem 5.1. Assume that $\mu$ is a Radon probability measure on $S M$ such that $\mu$ is invariant under the geodesic flow and $I_{\alpha}(\mu)<\infty$ for $\alpha>2$. Let $\gamma<(\alpha-2) / 2$.

By Lemma 3.2 we may restrict our consideration to the measures $\mu_{\delta}=\widetilde{P}_{*}\left(\nu \times \rho \mathcal{L}^{1}\right)$ where $\delta>0$ and $\rho$ is a smooth function such that spt $\rho \subset(0,1)$ and $\rho(t)=1$ for all $\delta<t<1-\delta$. Letting $n, k \in \mathbb{N}$ such that $\alpha>2+2 \gamma+1 / n$ and $k>n(1+2 \gamma+1 / n)$, and using Lemma 5.5 and Corollary 5.7 for positive $j$, we have

$$
\begin{aligned}
& \int_{\mathbb{R}^{2}}\left|\widehat{\mu_{\delta}}(\xi)\right|^{2}|\xi|^{2 \gamma} d \mathcal{L}^{2}(\xi) \leq C \sum_{j=-\infty}^{\infty} 2^{2 j \gamma} \int_{\mathbb{R}^{2}}\left(\psi_{2^{-j}} * \mu_{\delta}\right)(x) d \mu_{\delta}(x) \\
& \leq C \sum_{j=-\infty}^{\infty} 2^{2 j \gamma+2 j \mid} \mid \int_{\mathbb{R}^{2}} \int_{\mathbb{R}^{2}} \psi\left(2^{j}(x-y)\right) \\
& \times d \widetilde{P}_{*}\left(\nu \times \rho \mathcal{L}^{1}\right)(x) d \widetilde{P}_{*}\left(\nu \times \rho \mathcal{L}^{1}\right)(y) \mid \\
& =C \sum_{j=-\infty}^{\infty} 2^{2 j \gamma+2 j \mid} \int_{\widetilde{I}^{2} \times \mathbb{R}} \int_{\widetilde{I}^{2} \times \mathbb{R}} \rho(a) \rho(b) \psi\left(2^{j}\left(P_{a}(q)-P_{b}\left(q^{\prime}\right), a-b\right)\right) \\
& \leq C \int_{\widetilde{I}^{2}} \int_{\widetilde{I}^{2}} \sum_{j=-\infty}^{\infty} 2^{2 j \gamma+2 j}\left(1+2^{j}\right)^{-\frac{n-1}{n}}\left(1+2^{j}\left|q-q^{\prime}\right|\right)^{-\frac{k}{n}} d \nu(q) d \nu\left(q^{\prime}\right) \\
& \leq C \int_{\widetilde{I}^{2}} \int_{\widetilde{I}^{2}}\left|q-q^{\prime}\right|^{-(1+2 \gamma+1 / n)} d \nu(q) d \nu\left(q^{\prime}\right)=C I_{1+2 \gamma+1 / n}(\nu) .
\end{aligned}
$$

Here the last inequality follows by picking a positive integer $j_{0}$ such that $2^{-j_{0}-1} \leq r<2^{-j_{0}}$ and by dividing the sum into 3 parts: $j<0$, $0 \leq j \leq j_{0}$, and $j>j_{0}$. Using the choice of $n$ and applying Lemma 5.2 gives the claim.

\section{Non-PRESERVATION OF HAUSDORFF DimENSiON IN HigheR DIMENSIONAL MANIFOLDS}

In this section we construct examples of (locally) invariant measures whose Hausdorff dimensions decrease under the projection onto the base manifold. Because of Remark 4.5 the following setting is natural for such examples.

Example 6.1. For any $n \geq 3$ there exist an $n$-dimensional compact Riemannian manifold $M$ and a measure $\mu$ on the unit tangent bundle $S M$ such that it is locally invariant and its Hausdorff dimension decreases under the projection $\Pi: S M \rightarrow M$.

In fact, let $M$ be the flat n-dimensional torus $[-1,2]^{n}$ and let $I^{n}=$ $[0,1]^{n} \subset M$. Using the notation of section 3 , we set

$$
\mathcal{C}_{1}:=I^{n-1} \times\{0\} \text { and } \mathcal{C}_{2}:=I^{n-1} \times\{1\},
$$


and define a diffeomorphism $\Psi: I^{n-1} \times I^{n-1} \times[0,1] \rightarrow \Psi\left(I^{n-1} \times I^{n-1} \times\right.$ $[0,1])$ by

$$
\Psi(x, y, t)=\left(\gamma_{p, q}\left(d_{M}(p, q) t\right), \gamma_{p, q}^{\prime}\left(d_{M}(p, q) t\right)\right)
$$

where $p=\left(x_{1}, \ldots, x_{n-1}, 0\right) \in \mathcal{C}_{1}, q=\left(y_{1}, \ldots, y_{n-1}, 1\right) \in \mathcal{C}_{2}$, and $\gamma_{p, q}$ is the unique shortest geodesic parametrized by the Riemannian arc length which connects $p$ and $q$. Taking any measure $\nu$ such that

$$
\text { spt } \nu \subset\left\{(x, y) \in I^{n-1} \times I^{n-1} \mid x_{n-1}=y_{n-1}=0\right\}
$$

and defining $\mu=\Psi_{*}\left(\nu \times \mathcal{L}^{1}\right)$, we have $\operatorname{dim}_{\mathrm{H}} \Pi_{*} \mu \leq n-1$ since $\Pi_{*} \mu$ is supported by the $(n-1)$-dimensional plane $\left\{\left(m_{1}, \ldots, m_{n}\right) \in I^{n} \mid\right.$ $\left.m_{n-1}=0\right\}$. Furthermore, $\mu$ is locally invariant, and $\operatorname{dim}_{\mathrm{H}} \mu=\operatorname{dim}_{\mathrm{H}} \nu+$ $1[\mathrm{H}]$. Choosing $\nu$ such that $\operatorname{dim}_{\mathrm{H}} \nu>n-2$ gives $\operatorname{dim}_{\mathrm{H}} \Pi_{*} \mu<\operatorname{dim}_{\mathrm{H}} \mu$.

Remark 6.2. (a) Example 6.1 is easily modified to verify the existence of a globally invariant measure whose Hausdorff dimension is not preserved when projecting onto the base manifold. To see this, take $\nu=\mathcal{L}^{2(n-2)}$ and replace $I^{n}$ by $M$ in Example 6.1. Then it is a straightforward calculation to show that $\operatorname{dim}_{\mathrm{H}}(\Pi \circ \psi)_{*}\left(\mathcal{L}^{2(n-2)} \times \mathcal{L}^{1}\right)=n-1$. Clearly, $\psi_{*}\left(\mathcal{L}^{2(n-2)} \times \mathcal{L}^{1}\right)=\mathcal{L}^{2 n-3}$ is globally invariant under the geodesic flow.

(b) In the case $n=3$ Example 6.1 may be reduced to the 2-dimensional case. Therefore we may apply the results of section 4 to deduce that

$$
\operatorname{dim}_{\mathrm{H}} \Pi_{*} \mu=\left\{\begin{array}{r}
\operatorname{dim}_{\mathrm{H}} \mu, \text { if } \operatorname{dim}_{\mathrm{H}} \nu \leq 1 \\
2, \text { if } \operatorname{dim}_{\mathrm{H}} \nu>1
\end{array}\right.
$$

\section{REFERENCES}

[FM] K. Falconer and P. Mattila, The packing dimension of projections and sections of measures, Math. Proc. Cambridge Philos. Soc., 119 (1996), 695713.

[H] H. Haase, On the dimension of product measures, Mathematika 37 (1990), 316-323.

[HT] X. Hu and J. Taylor, Fractal properties of products and projections of measures in $\mathbb{R}^{n}$, Math. Proc. Cambridge Philos. Soc. 115 (1994), 527-544.

[HK1] B.R. Hunt and V.Yu. Kaloshin, How projections affect the dimension spectrum of fractal measures?, Nonlinearity 10 (1997), 1031-1046.

[HK2] B.R. Hunt and V.Yu. Kaloshin, Regularity of embeddings of infinitedimensional fractal sets into finite-dimensional spaces, Nonlinearity $\mathbf{1 2}$ (1999), 1263-1275.

[JJL] E. Järvenpää, M. Järvenpää and M. Llorente, Local dimensions of sliced measures and stability of packing dimensions of sections of sets, to appear in Adv. Math.

[K] R. Kaufmann, On Hausdorff dimension of projections, Mathematika 15 (1968), 153-155.

[LL] F. Ledrappier and E. Lindenstrauss, On the projections of measures invariant under the geodesic flow, IMRN, 9 (2003), 511-526.

[Mar] M. Marstrand, Some fundamental geometrical properties of plane sets of fractional dimension, Proc. London Math. Soc. (3) 4 (1954), 257-302. 
[Mat1] P. Mattila, Hausdorff dimension, orthogonal projections and intersections with planes, Ann. Acad. Sci. Fenn. Math. 1 (1975), 227-244.

[Mat2] P. Mattila, Orthogonal projections, Riesz capacities and Minkowski content, Indiana Univ. Math. J. 39 (1990), 185-198.

[Mat3] P. Mattila, Geometry of Sets and Measures in Euclidean Spaces: fractals and rectifiability, Cambridge University Press, Cambridge, 1995.

[Mat4] P. Mattila, Hausdorff dimension, projections, and Fourier transform, to appear in Publ. Mat.

[PS] Y. Peres and W. Schlag, Smoothness of projections, Bernoulli convolutions, and the dimensions of exceptions, Duke Math. J. (2) 102 (2000), 193-251.

[SY] T. D. Sauer and J. A. Yorke, Are dimensions of a set and its image equal for typical smooth functions?, Ergodic Theory Dynam. Systems (4) 17 (1997), 941-956.

Department of Mathematics and Statistics, P.O. Box 35, FIN-40014 UNIVERSITY OF JYVÄSKYLÄ, FINLAND ${ }^{1,2,3}$

E-mail address: esaj@maths.jyu.fi ${ }^{1}$

E-mail address: amj@maths.jyu.fi ${ }^{2}$

E-mail address: mileikas@maths.jyu.fi ${ }^{3}$ 